\title{
AN EFFICIENT ALGORITHM FOR GRAPH LAPLACIAN OPTIMIZATION BASED ON EFFECTIVE RESISTANCES
}

\author{
Eduardo Pavez and Antonio Ortega \\ University of Southern California, Los Angeles, California, USA
}

\begin{abstract}
In graph signal processing, data samples are associated to vertices on a graph, while edge weights represent similarities between those samples. We propose a convex optimization problem to learn sparse well connected graphs from data. We prove that each edge weight in our solution is upper bounded by the inverse of the distance between data features of the corresponding nodes. We also show that the effective resistance distance between nodes is upper bounded by the distance between nodal data features. Thus, our proposed method learns a sparse well connected graph that encodes geometric properties of the data. We also propose a coordinate minimization algorithm that, at each iteration, updates an edge weight using exact minimization. The algorithm has a simple and low complexity implementation based on closed form expressions.
\end{abstract}

Index Terms - effective resistance, graph learning, graph Laplacian, sparsity, matrix tree theorem

\section{INTRODUCTION}

Graphs are versatile tools for modeling high dimensional data and have been applied to data processing problems in various fields [1, $2,3,4]$. Graph nodes represent variables or features, and edges represent dependencies between them. In this paper we are interested in learning, from data, graphs that are both sparse and well connected. Sparse graphs, i.e., those where the number of edges is in the order of the number of nodes, are desirable because they lead to graph based algorithms, e.g., graph filters [4], that have lower computational complexity and are easier to interpret. However, sparse graphs are more likely to be disconnected, which can be undesirable, since well connected graphs lead to graph algorithms that have higher performance, robustness and adapt better to local geometry $[5,6,7,8,9,10,11]$.

There are various ways of quantifying how well connected a graph is. Some authors define well connected graphs as having small total resistance [9, 12], while others have defined them as having large algebraic connectivity [13]. These quantities have been used to characterize the rate of information propagation [5, 6], and communication among agents in a network [7, 8]. A third approach is based on the matrix-tree theorem, which states that the number of spanning trees of a graph is equal to a minor (determinant of a certain sub-matrix) of the Laplacian. Under these metrics, connectedness can be improved by adding edges or by increasing edge weights.

Graph learning algorithms select a graph topology (edge set) and edge weights to capture similarity between data points (features) associated to nodes. Conventional data driven methods compute pairwise distances between nodes, based on their respective features, and

This work was funded in part by NSF under grant CCF-1410009, and by a Google Faculty Research Award select a graph topology based on these distances, e.g., by connecting nodes to their $K$ nearest neighbors. Edge weights are assigned inversely proportional to the distance, e.g., using a kernel similarity.

We take a more general approach and compute a cost $h_{i j}$ which can be any dissimilarity (distance-like function) between features at nodes $i$ and $j$, e.g., the distance used in traditional approaches. The goal of these costs is to encourage smaller (or zero) weights, when nodes in the graph are expected to be dissimilar. Our graph learning algorithm follows two steps. First we choose a sparse graph topology based on the edge costs, e.g., by inputting them to a $K$ nearest neighbors algorithm. We provide a theoretical justification for distance based graph topology selection in Section 3. Second, we optimize the edge weights while guaranteeing that the resulting graph remains well connected. This is done by using a convex negative log determinant objective function, for which minimization is equivalent to maximizing the Laplacian minor (Section 4).

Early work to learn sparse well connected graphs jointly optimized the topology and edge weights without using data [13, 12]. More recently, [14] and others (see references therein), start from a weighted complete graph, and maximize graph connectivity (using the Laplacian minor) under sparsity constraints. These approaches optimize the graph topology, and although the initial edge weights can be data dependent, they are not changed (optimized) by the algorithm. Others $[15,16,17,18]$ have proposed smoothness based graph learning problems, that lead to data adaptive sparse graphs. If the optimization problem only included a smoothness objective function, the optimal solution would be a graph with all edge weights equal to zero. To avoid these trivial solutions, additional constraints and regularization terms that penalize graphs with isolated nodes and small weights can be introduced $[15,16,17,18]$. However, the resulting graphs might still have disconnected components, and choosing a good set of parameters that balances sparsity, connectedness and data fidelity becomes an additional burden.

Our first contribution is a theoretical analysis of the case when the input graph topology is fixed but otherwise arbitrary (e.g., it could be the complete graph). We show that for a particular choice of edge costs (see Section 2), the proposed optimization problem is equivalent to estimation of attractive intrinsic Gaussian Markov random fields (GMRF) [3]. This problem was initially studied in [19] as an extension of the graphical Lasso [20], where the precision matrix is the Laplacian. We prove two important properties of this optimal graph that are related to connectedness and sparsity (Proposition 1). First, we show that the effective resistance distance [21] of an edge is upper bounded by the edge cost, thus enforcing that similar nodal features (small cost) are mapped to nearby graph nodes. Second, the magnitude of the optimal edge weight is bounded by the inverse of the edge cost, thus ensuring far apart data features, are related through a small edge weight in the optimal graph. Although several papers have studied this solution from probabilistic and algorithmic perspectives [19, 22, 23, 24], the derived bounds are new and provide 
insights into the geometric properties of the solution. In particular, these properties justify the use of distance based graph topology selection algorithms (e.g., $K$ nearest neighbors) when optimizing the Laplacian minor.

Our second contribution is a coordinate minimization algorithm to learn the edge weights. We obtain a closed form expression for each update that depends on the edge's effective resistance and cost. By exploiting some results on learning trees [25], we show that the proposed method can be implemented with $\mathcal{O}\left(n^{2}\right)$ complexity per iteration. The complexity is lower because we avoid a naive implementation that takes $\mathcal{O}\left(n^{3}\right)$ time per iteration. Compared to the first algorithm for this problem [19], and more recent improvements and extensions [22, 23, 24], our algorithm has lower complexity per iteration. In addition, once the data (in the form of edge costs), edge set $\mathcal{E}$, and a convergence tolerance are chosen, the proposed algorithm has no other parameters. Our code is available online ${ }^{1}$.

The rest of this paper is organized as follows. In Section 2 we introduce the graph learning problem and some graph theoretic concepts. Section 3 is concerned with theoretical properties of the optimal graph Laplacian. Section 4 introduces the proposed algorithm, while Section 5 provides numerical evaluation. Section 6 provides some conclusions and future research directions.

\section{PRELIMINARIES}

\subsection{Graph theoretic background}

We denote scalars, vectors and matrices using lowercase regular, lowercase bold, and uppercase bold font respectively, e.g., $a$, a, and $\mathbf{A}$. The symbols $\dagger$ and ${ }^{\top}$ indicate Moore-Penrose pseudo inverse and transpose respectively. The all ones $n \times n$ matrix is denoted by $\mathbf{J}_{n}$. We consider undirected connected graphs with positive weights and without self loops. A graph $\mathcal{G}=(\mathcal{V}, \mathcal{E}, \mathbf{W})$ with node set $\mathcal{V}=\{1, \cdots, n\}$, edge set $\mathcal{E} \subset \mathcal{V} \times \mathcal{V}$ has individual edges denoted by $e=(i, j) \in \mathcal{E}$, with corresponding edge weight $w_{e}=w_{i j}$. The weight matrix $\mathbf{W}=\left(w_{i j}\right)$ is symmetric and non negative, and $w_{i j}>0$ if and only if $(i, j) \in \mathcal{E}$ or $(j, i) \in \mathcal{E}$. Since we consider undirected graphs, we do not repeat edges when listing them, that is, if $(i, j) \in \mathcal{E}$ we do not include $(j, i)$ in the edge set. We also denote by $\mathbf{w}$, the $m$ dimensional vector with entries $w_{e}$ for $e \in \mathcal{E}$. The degree matrix is $\mathbf{D}=\operatorname{diag}(\mathbf{W} 1)$. For an edge $e=(i, j)$, the edge vector is denoted by $\mathbf{g}_{e} \in \mathbb{R}^{n}$, which has entries $\mathbf{g}_{e}(i)=1, \mathbf{g}_{e}(j)=-1$, and zero otherwise. The incidence matrix is $\boldsymbol{\Xi}=\left[\mathbf{g}_{e_{1}}, \mathbf{g}_{e_{2}}, \cdots, \mathbf{g}_{e_{m}}\right]$, while the combinatorial Laplacian matrix is defined as $\mathbf{L}=\mathbf{D}-\mathbf{W}=\boldsymbol{\Xi} \operatorname{diag}(\mathbf{w}) \boldsymbol{\Xi}^{\top}$. The strengthened Laplacian is defined as $\mathbf{Q}=\mathbf{L}+\frac{1}{n} \mathbf{J}_{n}$ [22]. The set of combinatorial Laplacian matrices of graphs with $n$ nodes is denoted by $\mathbb{L}_{n}$. The eigenvalues of $\mathbf{L}$ are denoted by $0=\lambda_{1} \leq \lambda_{2} \leq, \cdots, \leq \lambda_{n}$. To determine when a graph is well connected we define:

- A path from node $i$ to node $j$ is a sequence of edges $\mathcal{P}_{i j}=$ $\left\{\left(i_{1}, i_{2}\right), \cdots,\left(i_{t-1}, i_{t}\right)\right\} \subset \mathcal{E}$ so that $i=i_{1}$, and $j=i_{t}$.

- A graph is connected if $\forall(i, j) \in \mathcal{V} \times \mathcal{V}$, there exists $\mathcal{P}_{i j} \neq \emptyset$. A graph is connected if and only if $\lambda_{2}>0$, or if $\mathbf{Q} \succ 0$.

- A tree is a connected graph with $n-1$ edges.

- A spanning tree of $\mathcal{G}=(\mathcal{V}, \mathcal{E}, \mathbf{W})$ is any sub-graph with vertex set $\mathcal{V}$ that is a tree.

The weight of a spanning tree $\mathcal{T} \subset \mathcal{E}$ of graph $\mathcal{G}=(\mathcal{V}, \mathcal{E}, \mathbf{W})$ is the product of its edge weights, that is, $\Omega\left(\mathbf{L}_{\mathcal{T}}\right)=\prod_{e \in \mathcal{T}} w_{e}$, where

\footnotetext{
${ }^{1}$ https://github.com/STAC-USC/graph_learning_ CombLap
}

$\mathbf{L}_{\mathcal{T}}=\sum_{e \in \mathcal{T}} w_{e} \mathbf{g}_{e} \mathbf{g}_{e}^{\top}$ is the Laplacian of the tree. Define the sum of weights of all spanning trees of $\mathcal{G}$ with Laplacian $\mathbf{L}$ as:

$$
\Omega(\mathbf{L})=\sum_{\mathcal{T} \subset \mathcal{E}} \Omega\left(\mathbf{L}_{\mathcal{T}}\right)
$$

Note that for unweighted graphs (all $w_{e}=1$ ) all trees have unit weight, hence $\Omega(\mathbf{L})$ is the number of spanning trees. Also note that for disconnected graphs $\Omega(\mathbf{L})$ is zero. Graphs with larger $\Omega(\mathbf{L})$ have more spanning trees of larger weights, so we can use this quantity to quantify how well connected a graph is. Based on (1) a graph connectivity can increase by adding edges (more spanning trees) or by increasing weights for existing edges (of existing spanning trees). Computing $\Omega(\mathbf{L})$ from its definition would require enumerating all spanning trees, fortunately there is an elegant alternative.

Theorem 1 (Matrix tree theorem). $\Omega(\mathbf{L})$ can be computed as:

$$
\Omega(\mathbf{L})=\frac{1}{n} \operatorname{det}\left(\mathbf{L}+\frac{1}{n} \mathbf{J}_{n}\right)=\frac{1}{n} \prod_{i=2}^{n} \lambda_{i} .
$$

For any pair of vertices $i$ and $j$, their effective resistance is:

$$
r_{i j}=r_{e}=\mathbf{g}_{e}^{\top} \mathbf{L}^{\dagger} \mathbf{g}_{e}=\mathbf{g}_{e}^{\top} \mathbf{Q}^{-1} \mathbf{g}_{e},
$$

where $e=(i, j) \in \mathcal{V} \times \mathcal{V} . r_{i j}$ obeys the triangular inequality and other metric properties [21], thus nodes $i$ and $j$ are well connected when $r_{i j}$ is small. Since $\log \operatorname{det}(\mathbf{Q})=\log (n \Omega(\mathbf{L}))$, we have that

$$
\frac{\partial \log \operatorname{det}(\mathbf{Q})}{\partial w_{e}}=\operatorname{tr}\left(\mathbf{Q}^{-1} \frac{\partial \mathbf{Q}}{\partial w_{e}}\right)=\operatorname{tr}\left(\mathbf{Q}^{-1} \mathbf{g}_{e} \mathbf{g}_{e}^{\top}\right)=r_{e}
$$

\subsection{Edge cost}

Data is available in matrix $\mathbf{X} \in \mathbb{R}^{n \times N} \cdot \mathbf{x}^{(k)}$ is the $k$-th row of $\mathbf{X}$, and corresponds to the data point (feature) associated to the $k$-th node. Alternatively, the $k$-th column of $\mathbf{X}$ is denoted by $\mathbf{x}_{k}$, which can be viewed as a graph signal. We define $h_{e}$ as the quantity that represents the cost of including edge weight $e=(i, j)$ in the graph. Intuitively, edges with small costs are more likely to be given larger weights. Examples of edge costs are given next.

Estimation of attractive intrinsic Gaussian Markov random fields (GMRF). If the edge cost is chosen as

$$
h_{e}=h_{i j}=\alpha+\frac{1}{N} \sum_{k=1}^{N}\left(x_{i k}-x_{j k}\right)^{2},
$$

then $\sum_{e} h_{e} w_{e}=\operatorname{tr}(\mathbf{L S})+\alpha\|\mathbf{w}\|_{1}$, where $\mathbf{S}=\frac{1}{N} \mathbf{X X}^{\top}$. This cost term appears in estimation of sparse GMRFs that have a precision matrix in the form of a Laplacian [19, 22, 23], under the assumption that $\mathbf{x}_{k}$ are zero mean independent identically distributed graph signals.

Learning graphs from smooth signals. For any $p>0$, the $\ell_{p}$ Laplacian variation of a signal $\mathbf{x}$ is $V_{p}(\mathbf{x})=\sum_{(i, j) \in \mathcal{E}} w_{i j}\left|x_{i}-x_{j}\right|^{p}$. The average variation of the columns of $\mathbf{X}$ is

$\frac{1}{N} \sum_{k=1}^{N} V_{p}\left(\mathbf{x}_{k}\right)=\sum_{(i, j) \in \mathcal{E}} w_{i j} \frac{1}{N} \sum_{k=1}^{N}\left|x_{i k}-x_{j k}\right|^{p}=\sum_{(i, j) \in \mathcal{E}} w_{i j} h_{i j}$.

Recently, $[15,16,18]$ used this criterion to learn sparse graphs.

Similarity graph optimization. Note that the costs in (3) and (4) are distances between the rows of $\mathbf{X}$. We can choose any distance-like function, for example a Gaussian kernel cost

$$
h_{i j}=\exp \left(\left\|\mathbf{x}^{(i)}-\mathbf{x}^{(j)}\right\|^{2} / \sigma^{2}\right) .
$$




\section{PROBLEM FORMULATION AND PROPERTIES}

In this section we state the graph learning problem and establish some properties of the solution. We divide the problem into two steps: edge set selection, and edge weight estimation. Edge weights are obtained by solving the optimization problem

$$
\min _{\mathbf{L} \in \mathbb{L}_{n}}-\log \operatorname{det}(\mathbf{Q}) \text { s.t. }, \quad w_{e}=0, e \notin \mathcal{E} \quad \sum_{e \in \mathcal{E}} h_{e} w_{e} \leq C .
$$

Note that the negative log determinant is convex, and ensures the graph is connected. Without loss of generality we pick $C=n-1$.

In (6), we assume that the edge set $\mathcal{E}$ is given. If no prior information is available, $\mathcal{E}$ can be chosen as the complete graph. Second, we address the problem of choosing an edge set $\mathcal{E}$ that is sparse, i.e., it has at most $m=\mathcal{O}(n)$ edges. Our approach is based on a theoretical analysis of the solution of (6) when $\mathcal{E}$ is the complete graph. The Lagrangian of (6) is

$-\log \operatorname{det}(\mathbf{Q})+\nu\left(\sum_{e \in \mathcal{E}} h_{e} w_{e}-(n-1)\right)-\sum_{e \in \mathcal{E}} \lambda_{e} w_{e}-\sum_{e \notin \mathcal{E}} \gamma_{e} w_{e}$.

Then, the Karush Kuhn Tucker (KKT) optimality conditions are,

$$
\begin{aligned}
& -r_{e}+\nu h_{e}-\lambda_{e}=0, \quad \lambda_{e}, w_{e} \geq 0, \quad \lambda_{e} w_{e}=0, \quad \forall e \in \mathcal{E}, \\
& \sum_{e \in \mathcal{E}} h_{e} w_{e} \leq n-1, \quad \nu\left(\sum_{e \in \mathcal{E}} h_{e} w_{e}-(n-1)\right)=0, \quad \nu \geq 0, \\
& -r_{e}-\gamma_{e}=0, \quad \forall e \notin \mathcal{E} .
\end{aligned}
$$

Any connected graph obeys $\sum_{e} r_{e} w_{e}=n-1$, which combined with the KKT conditions implies that $\nu=1$ and $\sum_{e} w_{e} h_{e}=n-1$. After further algebraic manipulations we obtain the following.

Proposition 1. The effective resistances of the solution of (6) obey

$$
r_{e}^{*}=h_{e}, \forall e \in \mathcal{E}^{*}, \quad r_{e}^{*} \leq h_{e}, \forall e \in \mathcal{E} \backslash \mathcal{E}^{*},
$$

while the weights satisfy

$$
w_{e}^{*} \leq 1 / h_{e}, \text { if } e \in \mathcal{E}^{*} .
$$

These bounds explicitly associate the data similarity to the connectedness and sparsity of the graph through the effective resistance and graph weights, respectively. When features of nodes $i$ and $j$ are close, i.e., $h_{i j}$ is small, the optimal edge will have a low effective resistance, thus ensuring nearby nodes are well connected in the graph. On the other hand, when $h_{i j}$ is large, the corresponding optimal weight is close to zero. Similar bounds based on correlation instead of distance are available when the goal is learning a generalized graph Laplacian [26, Ch. 4].

An important consequence of Proposition 1 is that it reveals information about the optimal graph, without having to solve (6). Since an edge with large costs $h_{i j}$ will have zero or small weights, a sparse edge set $\mathcal{E}$ can be chosen as the subset of the complete graph, for which the pairs $(i, j)$ have a small edge cost. We illustrate this numerically in Section 5, using $K$ nearest neighbor algorithm.

\section{COORDINATE MINIMIZATION ALGORITHM}

It can be proven that the solution of (6) is equal to the solution of

$$
\min _{\mathbf{L}} F(\mathbf{L}) \quad \text { s.t. } w_{e} \geq 0, e \in \mathcal{E}, \quad w_{e}=0, e \notin \mathcal{E},
$$

where $F(\mathbf{L})=-\log \operatorname{det}(\mathbf{Q})+\sum_{e} h_{e} w_{e}$. In this section we derive an iterative algorithm for solving (9), that at each step solves

$$
w_{e}^{(t+1)}=\underset{w_{e} \geq 0}{\arg \min } F(\mathbf{L}) \text { s.t. } w_{f}=w_{f}^{(t)}, \forall f \neq e,
$$

for $e \in \mathcal{E}$. This type of algorithm is known as coordinate minimization. For general convex optimization problems, one can solve (10) using coordinate descent with line search, which can be computationally expensive since it requires multiple evaluations of the objective function. For this problem, however, there is a closed form solution. Let $\mathbf{L}^{(t)}$, and $\mathbf{Q}^{(t)}$ be the estimates at the $t$-th iteration, so that $\mathbf{L}^{(t)}+(1 / n) \mathbf{J}_{n}=\mathbf{Q}^{(t)}$. The update of the graph Laplacian is

$$
\mathbf{L}^{(t+1)}=\mathbf{L}^{(t)}+\delta_{e}^{(t+1)} \mathbf{g}_{e} \mathbf{g}_{e}^{\top} .
$$

where $\delta_{e}^{(t+1)}=w_{e}^{(t+1)}-w_{e}^{(t)}$. Our main result is stated below.

Theorem 2. If $\mathbf{L}^{(t)}$ is irreducible, then (10) is solved by

$$
w_{e}^{(t+1)}=\max \left(0, w_{e}^{(t)}+1 / h_{e}-1 / r_{e}^{(t)}\right),
$$

and the updated Laplacian $\mathbf{L}^{(t+1)}$ is also irreducible.

Proof. The update (11) changes the determinant as follows

$$
\operatorname{det}\left(\mathbf{Q}^{(t)}+\delta_{e}^{(t+1)} \mathbf{g}_{e} \mathbf{g}_{e}^{\top}\right)=\left(1+\delta_{e}^{(t+1)} r_{e}^{(t)}\right) \operatorname{det}\left(\mathbf{Q}^{(t)}\right) .
$$

The change in the objective function is

$$
F\left(\mathbf{L}^{(t+1)}\right)-F\left(\mathbf{L}^{(t)}\right)=-\log \left(1+\delta_{e}^{(t+1)} r_{e}^{(t)}\right)+\delta_{e}^{(t+1)} h_{e} .
$$

(10) can be solved by minimizing (14) subject to $w_{e} \geq 0$. The optimality conditions of that problem are

$$
\frac{-r_{e}^{(t)}}{1+\delta_{e}^{(t+1)} r_{e}^{(t)}}+h_{e}-\lambda_{e}=0, \lambda_{e}, w_{e}^{(t+1)} \geq 0, \lambda_{e} w_{e}^{(t+1)}=0
$$

which are satisfied by (12). Note that $\mathbf{L}^{(t+1)}$ is irreducible if and only if the corresponding graph associated to its non zero pattern is connected. This is equivalent to $\mathbf{Q}^{(t+1)} \succ 0$. Since $\mathbf{Q}^{(t+1)}$ is positive semi-definite by construction, we only have to prove that it is also non-singular. Using (13), and since we assume that $\operatorname{det}\left(\mathbf{Q}^{(t)}\right)>0$, we need to show that $1+r_{e}^{(t)} \delta_{e}^{(t+1)}>0$. (12) implies $\delta_{e}^{(t+1)} \geq 1 / h_{e}-1 / r_{e}^{(t)}$, which combined with $r_{e}^{(t)}>0$, and $h_{e}>0$, gives the desired result.

Coordinate minimization algorithm based on Theorem 2 can be implemented in $\mathcal{O}\left(n^{2}\right)$ time per edge weight update. Complexity is dominated by computation of the effective resistance. This and other issues related to computational complexity are discussed next.

Initialization. Theorem 2 is useful if we can find an initial estimate $\mathbf{L}^{(0)}$ that is irreducible, that is, its graph is connected and contained in $\mathcal{E}$. To find $\mathbf{L}^{(0)}$ we use the graph Laplacian that minimizes $F(\mathbf{L})$ over all spanning trees $\mathcal{T} \subset \mathcal{E}$. This problem was solved in [25], with complexity $\mathcal{O}(|\mathcal{E}| \log (n))$.

Computing effective resistances. All effective resistances of a tree can be computed in $\mathcal{O}\left(n^{2}\right)$ time using Gaussian elimination with a perfect elimination ordering [27]. For $t>0$, the matrix $\left(\mathbf{Q}^{(t+1)}\right)^{-1}$ can be updated using the Sherman-Morrison formula

$$
\left(\mathbf{Q}^{(t+1)}\right)^{-1}=\boldsymbol{\Sigma}^{(t+1)}=\boldsymbol{\Sigma}^{(t)}-\frac{\delta_{e}^{(t+1)}\left(\boldsymbol{\Sigma}^{(t)} \mathbf{g}_{e}\right)\left(\boldsymbol{\Sigma}^{(t)} \mathbf{g}_{e}\right)^{\top}}{1+\delta_{e}^{(t+1)} \mathbf{g}_{e}^{\top} \boldsymbol{\Sigma}^{(t)} \mathbf{g}_{e}}
$$




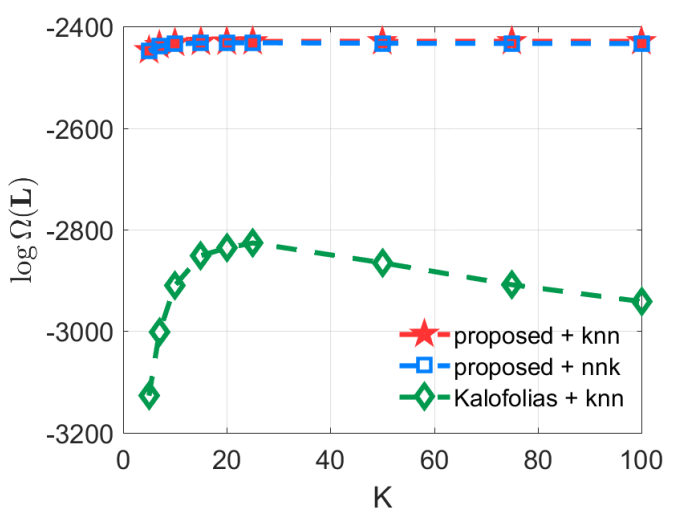

Fig. 1: Effect of graph topology inference on connectedness.

Given $e=(i, j)$ and $f=(s, t)$, the updated effective resistances is

$$
r_{f}^{(t+1)}=r_{f}^{(t)}-\left(\delta_{e}^{(t+1)} z_{e f}^{(t)}\right) /\left(1+\delta_{e}^{(t+1)} r_{e}^{(t)}\right)
$$

where $z_{e f}^{(t)}=\left(-r_{i s}^{(t)}+r_{i t}^{(t)}+r_{j s}^{(t)}-r_{j t}^{(t)}\right)^{2} / 4$. By keeping the matrix $\boldsymbol{\Sigma}^{(t)}$ in memory, all effective resistances can be computed in $\mathcal{O}\left(n^{2}\right)$ time after each weight update.

Edge selection rules. In theory, various rules lead to algorithms with the same convergence rate. However, greedy rules have been shown to provide great improvements for some cases [28]. We implement cyclic, random and a greedy rule. The cyclic rule iterates over edges $e \in \mathcal{E}$ in a fixed order. The randomized rule picks edges $e \in \mathcal{E}$ uniformly at random. Both have complexity $\mathcal{O}(1)$. Greedy rules pick the edge that maximizes a certain criteria. We use the proximal gradient Gauss-Southwell (PGS) rule, which chooses the edge with the largest $\Delta_{e}^{(t)}=\left|\delta_{e}^{(t)}\right|$. The PGS rule has complexity $\mathcal{O}(|\mathcal{E}|)$ per iteration.

Convergence. We say the coordinate minimization algorithm has converged if the decrease of the objective function after one epoch $(|\mathcal{E}|$ iterations $)$ is below a predefined threshold. There is no need to check feasibility, since the iterates are guaranteed by Theorem 2 to be connected. The initial value of the objective function $F\left(\mathbf{L}^{(0)}\right)$ can be computed in $\mathcal{O}(n)$ time [25]. For $t \geq 1$, we update the objective function using $(14)$ in $\mathcal{O}(1)$ time, therefore there is no additional computational burden to check feasibility or convergence.

\section{EXPERIMENTS}

To evaluate our theoretical results we learn graphs for the USPS handwritten digits [29]. We randomly select 100 images from each digit, to form a 1000 data set, and learn a $n=1000$ nodes similarity graph. Edge costs are computed using (5).

Graph topology inference. We study how sparsity of the input edge set $\mathcal{E}$ affects the learned graph. We consider two methods for choosing the graph topology. We use $K$ nearest neighbor graphs (KNN), where each node is connected to its $K$ closest points. We also use a method based on non negative kernel (NNK) regression [30], which is a low complexity algorithm that sparsifies KNN graphs based on local geometry. We compute the logarithm of (1) for the solution of (6) as a function of number of nearest neighbors $K$. The input graph topology $\mathcal{E}$ is obtained using the KNN and NNK algorithms. We compare against [16], since that algorithm also uses as input a KNN graph topology. Figure 1 indicates that the optimal

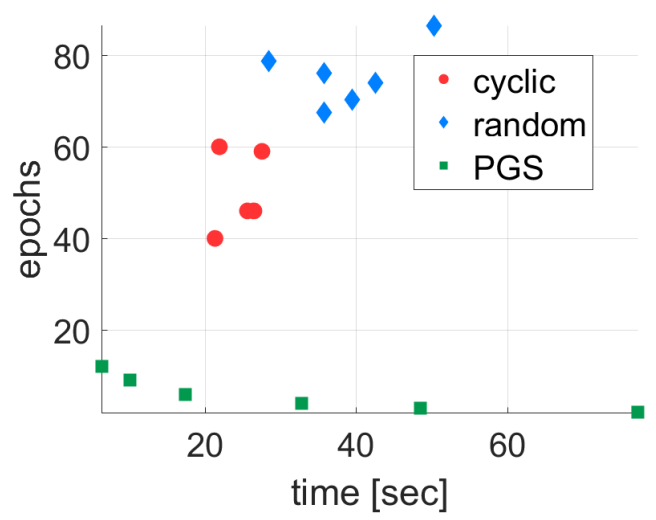

Fig. 2: Effect of edge selection rules on convergence.

graph Laplacian does not change when the input topology gets more dense (by increasing $K$ ). This can be explained by Proposition 1, which indicates that the magnitude of the optimal edge weights decays inversely proportional to the edge cost. The graph sparsity and density of edge weights becomes stable as $K$ increases. This indicates that in practice, a small value of $K$ can be chosen, which can have significant reduction on computational complexity (see Fig. 2). Another interesting observation is the fact that the NNK graph, even tough it is much sparser than the KNN graph (see [30]), produces a similar graph Laplacians. In contrast, the graph learning method from [16] returns a graphs whose connectedness decreases when the input topology becomes more dense.

Convergence and complexity of edge selection rules. We compare the cyclic, randomized and PGS edge selection for the same data set created for the previous experiment. We say that a algorithm has converged if after one epoch, the decrease in the objective function is smaller than $10^{-10}$. We choose edge sets using KNN with $K \in\{5,10,20,40,60,100\}$. The number of epochs versus convergence time are plotted in Figure 2. Using the cyclic rule lead to convergence in fewer iterations than using random edge selection. Also, as predicted by [28], coordinate minimization with the PGS rule converges is much fewer iterations than with the randomized and cyclic rules. On the other hand, the complexity per iteration grows proportionally with the edge density, thus the PGS rule should be used for sparse graphs, while the cyclic rule for more dense graph topologies.

\section{CONCLUSION}

We have analyzed a framework for learning combinatorial graph Laplacian matrices from data, with the goal of obtaining a sparse and well connected graph. Our theoretical analysis indicates that the optimal edge weights decay inversely proportional to the distance between nodal features, thus suggesting that distance like graph topology inference algorithms, such as KNN and others [30] can be used for edge set selection. We also propose a coordinate minimization algorithm that has low complexity per iteration thus it can be used to learn sparse graphs with hundreds to thousand of nodes. 


\section{REFERENCES}

[1] Ramón García-Domenech, Jorge Gálvez, Jesus V de JuliánOrtiz, and Lionello Pogliani, "Some new trends in chemical graph theory," Chemical Reviews, vol. 108, no. 3, pp. 11271169, 2008.

[2] Takayuki Ishizaki, Aranya Chakrabortty, and Jun-Ichi Imura, "Graph-theoretic analysis of power systems," Proceedings of the IEEE, vol. 106, no. 5, pp. 931-952, 2018.

[3] Havard Rue and Leonhard Held, Gaussian Markov random fields: theory and applications, CRC Press, 2005.

[4] Antonio Ortega, Pascal Frossard, Jelena Kovačević, José MF Moura, and Pierre Vandergheynst, "Graph signal processing: Overview, challenges, and applications," Proceedings of the IEEE, vol. 106, no. 5, pp. 808-828, 2018.

[5] Wei Ren, Randal W Beard, and Ella M Atkins, "A survey of consensus problems in multi-agent coordination," in American Control Conference, 2005. Proceedings of the 2005. IEEE, 2005, pp. 1859-1864.

[6] Reza Olfati-Saber and Richard M Murray, "Consensus problems in networks of agents with switching topology and timedelays," IEEE Transactions on automatic control, vol. 49, no. 9, pp. 1520-1533, 2004.

[7] Sebastian Thrun and Michael Montemerlo, "The graph slam algorithm with applications to large-scale mapping of urban structures," The International Journal of Robotics Research, vol. 25, no. 5-6, pp. 403-429, 2006.

[8] Kasra Khosoussi, Matthew Giamou, Gaurav S Sukhatme, Shoudong Huang, Gamini Dissanayake, and Jonathan P How, "Reliable graphs for slam," The International Journal of Robotics Research, vol. 38, no. 2-3, pp. 260-298, 2019.

[9] Changpeng Yang, Jianfeng Mao, Xiongwen Qian, and Peng Wei, "Designing robust air transportation networks via minimizing total effective resistance," IEEE Transactions on Intelligent Transportation Systems, 2018.

[10] Daniel A Spielman and Nikhil Srivastava, "Graph sparsification by effective resistances," SIAM Journal on Computing, vol. 40, no. 6, pp. 1913-1926, 2011.

[11] Joshua Batson, Daniel A Spielman, Nikhil Srivastava, and Shang-Hua Teng, "Spectral sparsification of graphs: theory and algorithms," Communications of the ACM, vol. 56, no. 8, pp. 87-94, 2013.

[12] Arpita Ghosh, Stephen Boyd, and Amin Saberi, "Minimizing effective resistance of a graph," SIAM review, vol. 50, no. 1, pp. 37-66, 2008.

[13] Arpita Ghosh and Stephen Boyd, "Growing well-connected graphs," in Decision and Control, 2006 45th IEEE Conference on. IEEE, 2006, pp. 6605-6611.

[14] H. Li, S. Patterson, Y. Yi, and Z. Zhang, "Maximizing the number of spanning trees in a connected graph," IEEE Transactions on Information Theory, pp. 1-1, 2019.

[15] X. Dong, D. Thanou, P. Frossard, and P. Vandergheynst, "Learning laplacian matrix in smooth graph signal representations," IEEE Transactions on Signal Processing, vol. 64, no. 23, pp. 6160-6173, Dec 2016.

[16] Vassilis Kalofolias, "How to learn a graph from smooth signals," in th International Conference on Artificial Intelligence and Statistics AISTATS, Cadiz, Spain, 2016, vol. 13.
[17] Samuel I Daitch, Jonathan A Kelner, and Daniel A Spielman, "Fitting a graph to vector data," in Proceedings of the 26th Annual International Conference on Machine Learning. ACM, 2009, pp. 201-208.

[18] Peter Berger, Manfred Buchacher, Gabor Hannak, and Gerald Matz, "Graph learning based on total variation minimization," in 2018 IEEE International Conference on Acoustics, Speech and Signal Processing (ICASSP). IEEE, 2018, pp. 6309-6313.

[19] Hilmi E Egilmez, Eduardo Pavez, and Antonio Ortega, "Graph learning from data under laplacian and structural constraints," IEEE Journal of Selected Topics in Signal Processing, vol. 11, no. 6, pp. 825-841, 2017.

[20] Jerome Friedman, Trevor Hastie, and Robert Tibshirani, "Sparse inverse covariance estimation with the graphical lasso," Biostatistics, vol. 9, no. 3, pp. 432-441, 2008.

[21] Wendy Ellens, FM Spieksma, P Van Mieghem, A Jamakovic, and RE Kooij, "Effective graph resistance," Linear algebra and its applications, vol. 435, no. 10, pp. 2491-2506, 2011.

[22] Sepideh Hassan-Moghaddam, Neil K Dhingra, and Mihailo R Jovanović, "Topology identification of undirected consensus networks via sparse inverse covariance estimation," in $D e$ cision and Control (CDC), 2016 IEEE 55th Conference on. IEEE, 2016, pp. 4624-4629.

[23] Licheng Zhao, Yiwei Wang, Sandeep Kumar, and Daniel P Palomar, "Optimization algorithms for graph laplacian estimation via ADMM and MM," IEEE Transactions on Signal Processing, vol. 67, no. 16, pp. 4231-4244, 2019.

[24] H. E. Egilmez, E. Pavez, and A. Ortega, "Graph learning from filtered signals: Graph system and diffusion kernel identification," IEEE Transactions on Signal and Information Processing over Networks, pp. 1-1, 2018.

[25] Keng-Shih Lu, Eduardo Pavez, and Antonio Ortega, "On learning laplacians of tree structured graphs," in 2018 IEEE Data Science Workshop (DSW). IEEE, 2018, pp. 205-209.

[26] Eduardo Pavez, Estimation of graph laplacian and covariance matrices, Ph.D. thesis, University of Southern California, 12 2019.

[27] Lieven Vandenberghe, Martin S Andersen, et al., "Chordal graphs and semidefinite optimization," Foundations and Trends $®$ in Optimization, vol. 1, no. 4, pp. 241-433, 2015.

[28] Julie Nutini, Mark Schmidt, Issam Laradji, Michael Friedlander, and Hoyt Koepke, "Coordinate descent converges faster with the gauss-southwell rule than random selection," in International Conference on Machine Learning, 2015, pp. 16321641.

[29] Yann LeCun, Bernhard E Boser, John S Denker, Donnie Henderson, Richard E Howard, Wayne E Hubbard, and Lawrence D Jackel, "Handwritten digit recognition with a back-propagation network," in Advances in neural information processing systems, 1990, pp. 396-404.

[30] Sarath Shekkizhar and Antonio Ortega, "Graph construction from data using non negative kernel regression (NNK graphs)," arXiv preprint arXiv:1910.09383, 2019. 